\title{
MASSIVE MACHINE-TYPE COMMUNICATIONS FOR IOT
}

Liang Liu, Erik G Larsson, Petar Popovski, Giuseppe Caire, Xiaoming Chen and Saeed R. Khosravirad

The self-archived postprint version of this journal article is available at Linköping University Institutional Repository (DiVA):

http://urn.kb.se/resolve?urn=urn:nbn:se:liu:diva-179629

N.B.: When citing this work, cite the original publication.

Liu, L., Larsson, E. G, Popovski, P., Caire, G., Chen, X., Khosravirad, S. R., (2021), MASSIVE MACHINE-TYPE COMMUNICATIONS FOR IOT, IEEE wireless communications, 28(4), 56-56. https://doi.org/10.1109/MWC.2021.9535445

Original publication available at:

https://doi.org/10.1109/MWC.2021.95354445

Copyright: Institute of Electrical and Electronics Engineers

http://www.ieee.org/index.html

(C)2021 IEEE. Personal use of this material is permitted. However, permission to reprint/republish this material for advertising or promotional purposes or for creating new collective works for resale or redistribution to servers or lists, or to reuse any copyrighted component of this work in other works must be obtained from the IEEE. 
The future wireless cellular networks are envisioned to not only enhance broadband access for humancentric applications, but also offer massive connectivity across tens of billions of devices for machinecentric applications empowered by the Internet of Things (IOT) technologies, e.g., smart factory and smart city. To embrace the forthcoming era of loT, the fifth-generation (5G) cellular communication standard has already identified massive machine-type communications (mMTC) as a key use case in future networks. Contrary to human-type high-speed communications, the accent of loT communications is on providing connectivity to tens of billions of devices with high energy efficiency, low latency, and high reliability. Consequently, the $5 \mathrm{G}$ and beyond cellular communication technologies call for radically innovative solutions to support mMTC. Motivated by the crucial role of MMTC in loT and the dramatically new challenges arising from mMTC as compared to human-type communication, this Special Issue (SI) aims to bring together researchers, industry practitioners, and individuals working on the related areas to share their new ideas, latest findings, and state-of-the-art results.

The article "Over-the-Air Computing for Wireless Data Aggregation in Massive loT" by Zhu et al., investigates wireless data aggregation for new loT applications such as distributed sensing, learning, and control. In particular, the authors leverage the over-the-air-computation (AirComp) technique, which turns the air into a computer for computing and communicating functions of distributed data at many devices by exploiting the waveform superposition property of multiple access channels. This article introduces several advanced AirComp techniques, including power control, MIMO AirComp, multi-cell AirComp, etc., to efficiently realize over-the-air aggregation of data simultaneously transmitted by devices.

The article "Deep Learning-Enhanced NOMA Transceiver Design for Massive MTC: Challenges, State-ofthe-Art, and Future Directions" by Ye et al., identifies non-orthogonal multiple access (NOMA) as a key enablers to meet various requirements in MMTC, considering the fact that a huge number of loT devices compete for the limited resources. The authors present a deep neural network (DNN) to unify the signal processing architecture and the multiuser receiver in both data and model-driven approaches, based on which the end-to-end communication is optimized. Then, the integration of non-orthogonal communication and neural computation is investigated to achieve high-performance communication with low cost.

The article "Massive Machine-Type Communication and Satellite Integration for Remote Areas" by Ullah et al., points out that remote areas are still poorly covered by the conventional terrestrial network due to the technical and economic reasons, and satellite network is an appealing solution to provide mMTC to the remote areas in the future. The authors propose a novel architecture for integrating low Earth orbit (LEO) satellites with the low-power wide-area network (LPWAN) technology and discuss the relevant changes for the protocol and how these can be addressed. Moreover, a simulation model is developed to obtain insight into the satellite-based LPWAN networks' performance limits and the respective trade-offs. 
The article "Machine Learning for Massive Industrial Internet of Things" by Zhou et al., focuses on a key use case of MMTC - industrial IoT (IloT). The authors first present several unique characteristics in IloT applications, which make it hard to directly apply the conventional machine learning technique to solve the practical problems, and then propose various solutions to embed these unique characteristics in the new machine learning design. Motivated by these solutions, several machine learning techniques are developed for the physical-layer design, the medium access control layer design, and the cross-layer design. Moreover, the authors present a case study of the massive access problem based on deep neural network and deep reinforcement learning techniques, respectively.

Biographies

Liang Liu is an assistant professor at The Hong Kong Polytechnic University (PolyU). He is the recipient of the 2017 IEEE Signal Processing Society Young Author Best Paper Award, and the best paper award for 2011 International Conference on Wireless Communications and Signal Processing (WCSP). He was listed in Highly Cited Researchers by Clarivate Analytics (Thomson Reuters) in 2018.

Erik G. Larsson is Professor at Linköping University, Sweden, and a Fellow of the IEEE. His research concentrates on physical-layer techniques for $5 \mathrm{G}$ and $6 \mathrm{G}$. He is highly cited according to ISI Web of Science, and received many awards for his work.

Petar Popovski is a Professor at Aalborg University. He received Dipl.-Ing. (1997)/Mag.-Ing. (2000) in communication engineering from Sts. Cyril and Methodius University in Skopje and Ph. D. from Aalborg University (2004). He is a Fellow of IEEE, holder of an ERC Consolidator Grant (2015-2020), Villum Investigator, and a Member at Large on the Board of Governors in IEEE Communication Society. His research interests are in wireless communications/networks and communication theory. He authored the book "Wireless Connectivity: An Intuitive and Fundamental Guide", published by Wiley in 2020.

Giuseppe Caire is currently an Alexander von Humboldt Professor with the Faculty of Electrical Engineering and Computer Science at the Technical University of Berlin, Germany. He received the Jack Neubauer Best System Paper Award from the IEEE Vehicular Technology Society in 2003, the IEEE Communications Society and Information Theory Society Joint Paper Award in 2004 and in 2011, the Okawa Research Award in 2006, the Alexander von Humboldt Professorship in 2014, the Vodafone Innovation Prize in 2015, an ERC Advanced Grant in 2018, the Leonard G. Abraham Prize for best IEEE JSAC paper in 2019, the IEEE Communications Society Edwin Howard Armstrong Achievement Award in 2020, and he is a recipient of the 2021 Leibinz Prize of the German National Science Foundation (DFG). Giuseppe Caire is a Fellow of IEEE since 2005. He has served in the Board of Governors of the IEEE Information Theory Society from 2004 to 2007, and as officer from 2008 to 2013. He was President of the IEEE Information Theory Society in 2011. 
Xiaoming Chen (IEEE Senior Member) is a Full Professor with Zhejiang University, China. He is currently serving as an Editor for the IEEE Transactions on Communications, and served as an Editor for the IEEE Communications Letters and a Guest Editor for the IEEE Journal on Selected Areas in Communications "Massive Access for 5 G and Beyond". He received the Best Paper Awards at IEEE/CIC ICCC 2018, IEEE ICC 2019, WCSP 2020, and IEEE GLOBECOM 2020.

Saeed R. Khosravirad is a Member of Technical Staff at Nokia Bell Labs. In this role, he contributes to innovating the future generation of wireless networks with ultrareliable and low latency access and joint communications and sensing capabilities towards enabling the wireless industrial revolution. He received his Ph.D. degree in telecommunications in 2015 from McGill University, Canada. He is an editor of the IEEE Transactions on Wireless Communications. 